\title{
Neuronal effects of nicotine during auditory selective attention
}

\author{
Jason Smucny, M.S. ${ }^{a, b, c}$, Ann Olincy, M.D. ${ }^{a, b}$, Lindsay C. Eichman, B.S. ${ }^{b}$, and Jason R. \\ Tregellas, Ph.D.

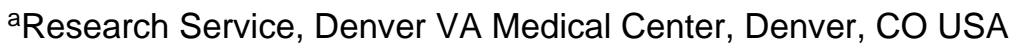 \\ bepartment of Psychiatry, University of Colorado Anschutz Medical Campus, Aurora CO USA \\ 'Neuroscience Program, University of Colorado Anschutz Medical Campus, Aurora CO USA
}

\begin{abstract}
Rationale-Although the attention-enhancing effects of nicotine have been behaviorally and neurophysiologically well-documented, its localized functional effects during selective attention are poorly understood.
\end{abstract}

Objectives-In this study, we examined the neuronal effects of nicotine during auditory selective attention in healthy human nonsmokers. We hypothesized to observe significant effects of nicotine in attention-associated brain areas, driven by nicotine-induced increases in activity as a function of increasing task demands.

Methods-A single-blind, prospective, randomized crossover design was used to examine neuronal response associated with a go/no-go task after $7 \mathrm{mg}$ nicotine or placebo patch administration in 20 individuals who underwent functional magnetic resonance imaging at 3T. The task design included two levels of difficulty (Ordered vs. Random stimuli) and two levels of auditory distraction (Silence vs. Noise).

Results-Significant treatment $\times$ difficulty $\times$ distraction interaction effects on neuronal response were observed in the hippocampus, ventral parietal cortex and anterior cingulate. In contrast to our hypothesis, $\mathrm{U}$ and inverted $\mathrm{U}$-shaped dependencies were observed between the effects of nicotine on response and task demands, depending on the brain area.

Conclusions-These results suggest that nicotine may differentially affect neuronal response depending on task conditions. These results have important theoretical implications for understanding how cholinergic tone may influence the neurobiology of selective attention.

\section{Keywords}

Attention; Auditory; fMRI; Nicotine; Parietal Cortex

Corresponding author: Jason Smucny (jason.smucny@ucdenver.edu).

The authors declare no conflicts of interest. 


\section{Introduction}

The potential utility of nicotinic agonists, including nicotine, as cognitive enhancers in health and disease necessitates their functional characterization in neuronal systems. Indeed, the cognitive-enhancing effects of nicotinic receptor targeting compounds are currently being investigated in Alzheimer's disease (Valles et al. 2014), autism (Ghaleiha et al. 2013), attention deficit (hyperactivity) disorder (Childress and Sallee 2014; Fleisher and McGough 2014), depression (Yu et al. 2014), schizophrenia (Freedman 2014), and healthy individuals (Demeter and Sarter 2013).

Among nicotine's pro-cognitive effects is its ability to improve performance during attention tasks (Kassel 1997; Levin et al. 1998; Newhouse et al. 2004; Stolerman et al. 1995). The construct of "attention" is complex. Three different forms of attention are commonly recognized: sustained attention, divided attention, and selective (or focused) attention. Sustained attention is the ability to maintain focus on stimuli for extended periods, divided attention is the ability to focus on more than one stimulus simultaneously, and selective attention is the ability to focus on one or more stimuli while ignoring others (Hahn et al. 2009). Nicotine has been shown to enhance behavioral performance and neurophysiological signatures associated with all three forms of attention, although the strongest evidence for behavioral improvement is during selective attention (Bain et al. 2003; Hahn et al. 2002; Kassel 1997). For example, nicotine improves performance on the Stroop Task, in which subjects are asked to focus on one characteristic of presented words (e.g. color or meaning) while ignoring the opposite characteristic (Poltavski and Petros 2006; Provost and Woodward 1991). Adding distracting stimuli enhances the attention-improving properties of nicotine (Grobe et al. 1998; Hahn et al. 2002) and nicotinic receptor subtype-specific agonists (Howe et al. 2010). Furthermore, in the auditory cortex, nicotine sharpens neuronal response to stimuli of target frequencies while suppressing response to unimportant frequencies (Metherate et al. 2012). Nicotine may enhance these processes by 1) increasing processing capacity, 2) increasing salience of relevant stimuli, 3) increasing arousal, and 4) decreasing processing of irrelevant stimuli, i.e. filtering (Kassel 1997). The relative contribution of each mechanism may depend on the task parameters. During easy tasks, for example, nicotine may increase processing capacity, allowing for more stimuli to be processed. Difficult tasks, on the other hand, may be more sensitive to nicotine's stimulusfiltering properties (Eysenck 1982; Kassel 1997).

The neurobiological systems associated with attention have been relatively wellcharacterized. It is well-established that two distinct attention systems exist in the brain: a dorsal attention network that includes the superior parietal lobule and frontal eye fields, and a ventral attention network that includes the ventral parietal cortex (VPC) (Corbetta and Shulman 2002, 2011). The dorsal attention network is involved in volitional, goal-driven, "top-down" control of attention, whereas the ventral network is important for stimulusdriven, sensory, "bottom-up" control of attention (Corbetta and Shulman 2002). Another "top down" brain area that may play an important role during attention is the anterior cingulate (AC), which is involved in regulating response conflict to "trigger strategic adjustments to cognitive control" (Botvinick et al. 2004). The AC is commonly recruited during tasks in which subjects are asked to inhibit prepotent responses (e.g. go/no-go tasks) 
(Botvinick et al. 2004). Previous imaging studies have found evidence for nicotinic modulation of both ventral and dorsal attention networks (Giessing et al. 2006; Kumari et al. 2003; Lawrence et al. 2002; Thiel and Fink 2008; Thiel et al. 2005). These studies have largely focused on sustained attention tasks in which researchers examined the effect of nicotine on task difficulty (e.g. Lawrence et al. 2002) or the effect of nicotine on valid vs. invalid attentional cuing (e.g. Thiel and Fink 2008; Thiel et al. 2005). The directionality of effects has been varied, with some studies showing recruitment of attention networks after nicotine administration (Kumari et al. 2003; Lawrence et al. 2002) and others showing decreased response (Thiel and Fink 2008; Thiel et al. 2005). These discrepancies may be due to differences in the subject population (smokers vs. non-smokers), drug dose, and/or task parameters (e.g. valid vs. invalid cuing, task difficulty). Related to this last point, according to the "attention allocation" model proposed by Kassel, nicotine may preferentially act as a stimulus enhancer or stimulus filter, depending on task conditions (Kassel 1997). The drug may therefore be expected to increase or decrease recruitment of attention-associated brain areas as a function of task demands.

Among the various forms of attention, nicotine's localized functional effects on selective attention may be the least extensively studied. In smokers, Gilbert et al. found that nicotine reduced the "distracting" effect of negative valence and smoking-related pictures as evidenced by stronger target stimulus-related event related potentials at parietal sites (Gilbert et al. 2007). Using fMRI, also in smokers, Hahn et al. (2009) observed a trend towards increased activation in frontal areas after nicotine administration (relative to placebo) during a visual selective attention task. A significant drug $\times$ task interaction was also observed, as nicotine decreased response in these areas during a simple target detection task. To our knowledge, however, no study has examined the localized neuronal effects of nicotine during auditory selective attention in the presence (or absence) of auditory distractors.

Although nicotine is a widely accepted attentional enhancer, additional whole brain human imaging studies are clearly needed to characterize its localized functional effects during selective attention, particularly in nonsmokers. To that end, the present study used fMRI to examine the functional circuits associated with nicotinic modulation of auditory selective attention in healthy nonsmoking subjects. In the attention task, subjects were asked to respond (button press) to auditory stimuli (single digit numbers other than " 3 ") under easy (numbers in order) or difficult (numbers presented in random order) in the presence (or absence) of environmental noise distractors. In accordance with the attention allocation model, we hypothesized to observe a significant drug $\times$ difficulty $\times$ distraction interaction in attention-associated brain areas such as the ventral parietal cortex. Furthermore, in accordance with this model, we hypothesized the interaction to be driven by increased neuronal response after nicotine administration (relative to placebo) as task demands increase. 


\section{Materials and Methods}

\section{Subjects}

Twenty healthy subjects participated in this study. Mean age was 37.35 (SD = 11.7), 9 females, 11 males, 18 right-handed, 2 left-handed. Subjects were excluded for a diagnosis of Axis I mental illness, first-degree family history of Axis I mental illness, neurological illness, head trauma, substance abuse, current (less than 3 months from last cigarette) smoking, poor (inability to hear $60 \mathrm{~dB} 1000$ and $1500 \mathrm{~Hz}$ tones in either ear) or unbalanced (> $10 \mathrm{~dB}$ threshold difference between each ear) hearing, failure to pass a physical examination, and functional magnetic resonance imaging (fMRI) exclusion criteria (claustrophobia, weight $>250 \mathrm{lbs}$, metal in the body). Mental illness, neurological illness, head trauma, substance abuse, and smoking status were assessed by informal interview. The study included 5 "former" (> 3 months from last cigarette) smokers. All subjects were required to pass a nicotine tolerance test, in which the nicotine dose used for the experiment $(7 \mathrm{mg}$ ) was administered $>3$ days prior to the first fMRI scan. Subjects provided written informed consent approved by the University of Colorado Institutional Review Board, and could withdraw from the study at any time. Subjects were compensated for participation.

\section{Study Design}

This was a single-blind, pseudo-randomized, placebo-controlled, crossover study. On each of two study visits, subjects were administered a $7 \mathrm{mg}$ nicotine patch (Nicoderm) or a placebo patch 70 minutes prior to fMRI scanning. Subjects wore patches throughout scanning. Total time of patch application was approximately 120 minutes $(70 \mathrm{~m}$ before scanning, $60 \mathrm{~m}$ during scanning). The attention task was performed during the first half of the scanning session. The placebo patch was tactilely and visually similar to the nicotine patch, although subjects were asked to refrain from examining either patch during or after application. The patch was removed immediately after scanning. Visits were scheduled $>3$ days apart. Heart rate and blood pressure were monitored immediately prior to patch application, 30 and 60 minutes after patch application, and up to 60 minutes after patch removal. Physiological effects of nicotine were analyzed using repeated measures analysis of variance (ANOVA) in SPSS20, with time (pretreatment vs. postreatment) and drug (placebo vs. nicotine) as within-subjects factors.

\section{Auditory Stimuli}

For the attention task (see "Task Description"), synthetic audio recordings for the numbers 1-9 were downloaded from www.modeltalker.com. Number stimuli were adjusted to have the same onset with Adobe Audition.

For task-overlaid noise distraction, environmental, "urban" noise stimuli were mixed as described previously (Tregellas et al. 2009). Briefly, clips included segments from two talk radio shows, two classical musical pieces, sounds from a neighborhood block party, which included multiple background conversations and sounds from children playing, traffic sounds, a refrigerator motor cycling on and off, and frequent knocking sounds from glasses being set on countertops. Volumes of all of these elements were mixed so that no one element was readily identifiable. The subjective experience of the sound mixture was that of 
standing in a busy crowd of people, in which multiple conversations were occurring, with a low level of indistinguishable background music and other sounds. Urban noise distraction was presented at $80 \mathrm{~dB}$ in the ear opposite the task-relevant stimuli with MR-compatible headphones (Resonance Technologies, Inc.).

\section{Task Description}

Subjects performed an auditory version of the Sustained Attention to Response Task (SART) (Seli et al. 2012). For the SART, single-digit numbers were aurally presented one at a time, and the subject was asked to respond (with a button press) (Lumina Response Pad, Cedrus Corp.) after each auditory stimulus ( $70 \mathrm{~dB}$, presented in either the right or left ear), except for the number ' 3 ,' in which case the subject was asked to withhold from responding. The ear (right or left) in which the numbers were presented was pseudo-randomized between subjects. Stimulus duration was $250 \mathrm{~ms}$ and inter-stimulus interval was $900 \mathrm{~ms}$. Subjects performed the Ordered SART and the Random SART. In the Ordered SART, the numbers were presented in order; in the Random SART, the numbers were presented pseudorandomly. Due to the predictability of Ordered SART, subjects may be able to correctly respond or withhold responding reflexively to the presence of any auditory stimulus. The unpredictability of Random SART, however, requires subjects to focus on specific stimulus features before making the appropriate response, increasing the task difficulty (Smucny et al. 2013). An identifier cue presented through MR-compatible goggles (Resonance Technologies, Inc.) outlined the current task condition (Ordered or Random) throughout the experiment. The identifier cue was presented $2.3 \mathrm{~s}$ before the first set of stimuli, as well $2.3 \mathrm{~s}$ before each time the condition switched from Ordered to Random (or vice-versa). The subject was asked to respond as quickly and accurately as possible to help induce attentiveness.

The task was presented as a block design, with four pseudo-randomly dispersed conditions: OrderedSilent (ordered numbers with no noise distraction), OrderedNoise (ordered numbers with noise distraction), RandomSilent (random numbers with no noise distraction), and RandomNoise (random numbers with noise distraction). 72 blocks of $12.65 \mathrm{~s}$ each were administered, with 18 blocks per condition. Each block consisted of 9-11 trials. Baseline data was collected from six 37.95 s fixation periods interspersed at regular intervals throughout the task.

Primary performance measures on the SART were 1) errors of commission, or incorrect button presses on ' 3 ', 2) errors of omission, or failure to button press on the numbers 1, 2, and 4-9, and 3) reaction time. Behavioral data were analyzed using repeated measures ANOVA in SPSS 20, with difficulty (Ordered vs. Random), distraction (Silence vs. Noise), and drug (placebo vs. nicotine) as within-subjects factors.

\section{fMRI Scanning Parameters}

Functional scans were collected using a clustered volume approach as described previously (Edmister et al. 1999; Tregellas et al. 2009). Use of the clustered volume approach allowed stimuli to be presented while minimizing scanner noise. This technique has been shown to 
substantially improve signal detection in fMRI experiments using auditory stimuli (Edmister et al. 1999).

Studies were performed with at 3T GE Signa MR system using a standard quadrature head coil. Functional images were acquired with a gradient-echo T2* Blood Oxygenation Level Dependent (BOLD) contrast technique, with $\mathrm{TR}=12650 \mathrm{~ms}$ (as a clustered volume acquisition of $2000 \mathrm{~ms}$, plus an additional $10650 \mathrm{~ms}$ silence interval), $\mathrm{TE}=30 \mathrm{~ms}$, FOV = $220 \mathrm{~mm}^{2}, 64^{2}$ matrix, 38 slices, $3.5 \mathrm{~mm}$ thick, $0.5 \mathrm{~mm}$ gap, angled parallel to the planum sphenoidale. Additionally, one IR-EPI (TI $=505 \mathrm{~ms})$ volume was acquired to improve spatial normalization (see "fMRI Data Analysis").

\section{fMRI Data Analysis}

Data were analyzed using SPM8 (Wellcome Dept. of Imaging Neuroscience, London). Data from each subject were realigned to the first volume, normalized to the Montreal Neurological Institute template, using a gray-matter-segmented inversion recovery echo planar image (IR-EPI) as an intermediate to improve coregistration between images (Anbeek et al. 2005) and smoothed with an 8-mm FWHM Gaussian kernel. A 196-s high pass filter was applied to remove low-frequency fluctuation in the BOLD signal.

To account for both within-group and within-subject variance, a whole-brain mixed effects analysis was implemented. Parameter estimates were generated for each individual in a firstlevel analysis. First-level effects were modeled with a double-gamma function, without temporal derivatives, using the general linear model in SPM8. For each treatment condition (placebo and nicotine), contrast images were generated for the four task conditions: OrderedSilent, OrderedNoise, RandomSilent, and RandomNoise. Fixation periods were used as an implicit baseline in order to ensure that an equivalent number of scans was used to model baseline as well as each condition (18 scans each). The resulting SPM contrast images were entered into a second-level, flexible factorial $2 \times 2 \times 2$ ANOVA in SPM8, with treatment, task difficulty (Ordered vs Random) and distraction level (Silent vs Noise) as within-subjects factors.

To test the hypothesis that nicotine (vs placebo) would differentially affect response according to task difficulty and distraction level, we used the following directional, interaction t-contrasts:

\section{Contrast 1:}

$\{$ Nicotine $(($ OrderedNoise $>$ OrderedSilent $)>($ RandomNoise $>$ RandomSilent $))\}>$ $\{$ Placebo $(($ OrderedNoise $>$ OrderedSilent $)>($ RandomNoise $>$ RandomSilent $))\}$

\section{Contrast 2}

$\{$ Nicotine $(($ RandomNoise $>$ RandomSilent $)>($ OrderedNoise $>$ OrderedSilent $))\}>$ $\{$ Placebo $(($ RandomNoise $>$ RandomSilent $)>($ OrderedNoise $>$ OrderedSilent $))\}$

The main effect of nicotine was analyzed using the contrast Nicotine > Placebo across all difficulty and distraction conditions. The main effect of difficulty was analyzed using the contrast Random > Ordered across all drug and distraction conditions. The main effect of 
distraction was analyzed using the contrast Noise $>$ Silent across all drug and difficulty conditions.

Contrasts of interest were evaluated at two significance thresholds. The most stringent threshold, $p<0.05$ FWE-corrected for multiple comparisons across the whole brain, found no significant clusters for any contrast of interest. The second, more lenient threshold was set at $p_{\text {corrected }}<.05$ based on 10,000 Monte Carlo simulations to correct for multiple comparisons based on cluster size. Precise estimates for Gaussian filter width were calculated by averaging estimated smoothness of residual images (ResMS.hdr files) generated from first-level analyses in SPM. Estimated average smoothness was $\mathrm{x}=9.34$ $\mathrm{mm}, \mathrm{y}=8.26 \mathrm{~mm}, \mathrm{z}=8.20 \mathrm{~mm}$. Based on these estimates, the simulated corrected threshold corresponded to a voxelwise threshold of $p<0.01$ and a minimum cluster size of 62 voxels.

After identifying all regions that displayed significant interactions under this threshold, peak GLM \% signal changes for each condition were extracted and averaged across group and/or condition for display purposes (Figures 1B, 1C, 2B, 2C, 3B, and 3C). To avoid any circular or non-independent analyses (Kriegeskorte et al. 2009), all post-hoc tests were performed in neuroimaging space using whole-brain corrected statistical thresholds and no secondary inferential statistical tests were performed on data extracted from these peaks. Extracted peak data presented in figures are non-independent and should not be used for effect-size estimates, but are included as a visual aid for the interpretation of significant interaction results from statistical analyses performed in neuroimaging space.

\section{Results}

\section{Physiological Effects of Nicotine}

Physiological effects of placebo vs. nicotine treatment are presented in Table 1.

Physiological data were not available from one subject due to an equipment malfunction. No significant time (pretreatment vs. $60 \mathrm{~m}$ post-treatment) $\times$ drug (placebo vs. nicotine) interactions were observed for systolic BP $(\mathrm{F}(1,18)=2.96, \mathrm{p}=0.10)$, diastolic $\mathrm{BP}(\mathrm{F}(1,18)=$ $1.27, \mathrm{p}=0.28)$, or heart rate $(\mathrm{F}(1,18)=1.44, \mathrm{p}=0.25)$, indicating that nicotine did not significantly affect any of these measures relative to placebo. Absence of a significant physiological effect of nicotine using the $7 \mathrm{mg}$ patch is consistent with previous observations (Poltavski and Petros 2005).

\section{Behavioral Results}

Behavioral data are presented in Table 2. No significant main effect of drug was observed for errors of commission $(F(1,19)=0.058, p=0.81)$, errors of omission $(F(1,19)=1.082, p$ $=0.31)$ or reaction time $(\mathrm{F}(1,19)=0.051, \mathrm{p}=0.82)$, indicating no significant behavioral effects of nicotine across all conditions. Significant main effects of difficulty (Ordered vs. Random) were observed for errors of commission $(F(1,19)=24.2$, $p<0.001)$ and reaction time $(\mathrm{F}(1,19)=50.3, \mathrm{p}<0.001)$ indicating significantly increased errors and slower performance under the Random condition. Significant main effects of distraction (Silence vs. Noise) were observed for errors of omission $(F(1,19)=10.3, p=0.005)$ and reaction time $(F(1,19)=13.9, p=0.001)$, indicating significantly increased errors and slower 
performance under distracting conditions. No significant drug $\times$ difficulty $\times$ distraction interaction was observed for errors of commission $(F(1,19)=0.096, p=0.76)$, errors of omission $(F(1,19)=0.18, p=0.68)$ or reaction time $F(1,19)=0.040, p=0.84)$.

\section{fMRI Results: Interaction Contrast \#1}

To test the hypothesis that nicotine (vs placebo) would differentially affect response according to task difficulty and distraction level, we used two directional interaction contrasts (see Methods). The first contrast found a significant interaction effect in the right hippocampus (peak z score $=3.62$, cluster size 91 voxels, peak coordinates $x=21, y=-28, z$ $=-11$, peak $\mathrm{p}<0.001 ;$ Figure 1A). The interaction was due to decreased response with nicotine during OrderedSilent, increased response during OrderedNoise, increased response during RandomSilent, and decreased response during RandomNoise (Figures 1B, 1C). Significant hippocampal response was not observed using post-hoc tests of simple main effects (Nicotine < Placebo for OrderedSilent and RandomNoise, Nicotine > Placebo for OrderedNoise and RandomSilent).

Due to the laterality of the hippocampus effect, the ANOVA analysis was repeated with handedness (left or right) as a covariate. Under these parameters, interactions remained significant (peak z score $=3.77$, cluster size $=106$ voxels, peak coordinates $x=21, y=-28$, $\mathrm{z}=-11$, peak $\mathrm{p}<0.001$ ). The ANOVA was also repeated using only right-handed subjects $(\mathrm{n}=18)$. This analysis also revealed significant interactions in the right hippocampus (peak $\mathrm{z}$ score $=3.77$, cluster size $=93$ voxels, peak coordinates $\mathrm{x}=21, \mathrm{y}=-28, \mathrm{z}=-11$, peak $\mathrm{p}<$ $0.001)$.

Due to the inclusion of former smokers, the ANOVA analysis was repeated with smoking status (never or former smoker) as a covariate. Under these parameters, a significant interaction effect was still observed in the right hippocampus (peak z score $=3.60$, cluster size $=102$ voxels, peak coordinates $\mathrm{x}=21, \mathrm{y}=-28, \mathrm{z}=-11$, peak $\mathrm{p}<0.001)$.

\section{fMRI Results: Interaction Contrast \#2}

The second interaction contrast (see Methods) yielded significant interaction effects in the left VPC (peak z score $=3.62$, cluster size $=96$ voxels, peak coordinates $\mathrm{x}=-63, \mathrm{y}=-52, \mathrm{z}$ $=25$, peak $\mathrm{p}<0.001$; Figure $2 \mathrm{~A}$ ) and $\mathrm{AC}$ (peak z score $=3.33$, cluster size $=207$ voxels, peak coordinates $\mathrm{x}=3, \mathrm{y}=11, \mathrm{z}=31$, peak $\mathrm{p}<0.001$; Figure $3 \mathrm{~A}$ ). The interaction was due to increased response after nicotine administration during the OrderedSilent condition, decreased response after nicotine administration during the OrderedNoise condition, decreased response after nicotine administration during the RandomSilent condition, and increased response after nicotine administration during the RandomNoise condition (Figures 2B, 2C, 3B, and 3C). Significant response was not observed in the VPC or AC using posthoc tests of simple main effects (Nicotine > Placebo for OrderedSilent and RandomNoise, Nicotine $<$ Placebo for OrderedNoise and RandomSilent).

Due to the laterality of the VPC effect, the ANOVA analysis was repeated with handedness (left or right) as a covariate. Under these parameters, a significant drug $\times$ difficulty $\times$ distraction was observed in the left VPC (peak z score $=3.60$, cluster size $=95$ voxels, peak coordinates $\mathrm{x}=-63, \mathrm{y}=-52, \mathrm{z}=25$, peak $\mathrm{p}<0.001$ ) but not the right VPC. The ANOVA 
was also repeated using only right-handed subjects $(n=18)$. This analysis revealed a significant drug $\times$ difficulty $\times$ distraction in the left VPC (peak z score $=3.83$, cluster size $=$ 142 voxels, peak coordinates $x=-63, y=-52, z=25$, peak $p<0.001$ ) but not the right VPC.

Due to the inclusion of former smokers, the ANOVA analysis was repeated with smoking status (never or former smoker) as a covariate. Under these parameters, significant interaction effects were still observed in the left VPC (peak z score $=3.61$, cluster size $=96$ voxels, peak coordinates $\mathrm{x}=-63, \mathrm{y}=-52, \mathrm{z}=25$, peak $\mathrm{p}<0.001$ ) and $\mathrm{AC}$ (peak z score $=$ 3.33 , cluster size $=210$ voxels, peak coordinates $\mathrm{x}=3, \mathrm{y}=11, \mathrm{z}=31$, peak $\mathrm{p}<0.001$ ).

\section{fMRI Results: Main Effects of Nicotine}

Brain regions in which nicotine was associated with increased response relative to placebo across all conditions are presented in Figure 4 and Table 3. Nicotine increased response in the primary motor cortex and supplementary motor cortices (Brodmann areas 4 and 6) as well as in the somatosensory cortex (Brodmann areas 2 and 3). Nicotine did not significantly decrease response relative to placebo in any area.

\section{fMRI Results: Main Effects of Difficulty}

Brain regions that showed a significant main effect of difficulty (Random > Ordered, across all other conditions) are presented in Table 4. Increased response was observed in the temporal gyrus, AC, superior frontal gyrus, inferior frontal gyrus, thalamus, and cerebellum.

Brain regions in which difficulty decreased response (Random < Ordered, across all other conditions) are presented in Table 5. Decreased response was observed in the medial frontal gyrus, subgenual cingulate, parahippocampal gyrus, precuneus, somatosensory cortex, and primary motor cortex, among other regions.

\section{fMRI Results: Main Effects of Distraction}

Significant main effects of distraction (Noise > Silence) were observed in the right temporal (auditory) cortex (peak z score $>8.2$, cluster size $=974$ voxels, peak coordinates $\mathrm{x}=-54, \mathrm{y}=$ $-13, \mathrm{z}=4$, peak $\mathrm{p}<0.001$ ) and the left temporal (auditory) cortex (peak z score $>8.2$, cluster size $=903$ voxels, peak coordinates $x=63, y=-7, z=4$, peak $p<0.001$ ). Noise distraction did not decrease response relative to Silence in any area.

\section{Discussion}

The primary goal of the present study was to test the hypothesis that nicotine would increase response during non-demanding conditions and decrease response during demanding task conditions in accordance with the model put forth by Kassel (1997). Task demands were manipulated through adjusting the level of auditory distraction and stimulus target predictability, and the hypothesis was tested using two directional interaction contrasts. This analysis yielded significant interaction effects in the hippocampus, VPC, and AC. As hypothesized, these findings suggest that nicotine may have differential effects on neuronal response depending on task conditions. The nature of nicotine's effects, however, did not support our hypothesis, in that nicotine had $\mathrm{U}$ and inverted-U shaped effects as a function of 
task demands. These results have interesting implications for understanding the relationships between task demands, neuronal response, and cholinergic tone.

Imaging results yielded significant interaction effects of nicotine in the VPC but not the dorsal parietal cortex, suggesting that nicotinic modulation in parietal areas may be preferential to the ventral, sensory-driven, "bottom up" stimulus attentional processing stream outlined by Corbetta and Shulman (2002). Nicotinic modulation of the VPC is consistent with previous studies during sustained and selective attention (Giessing et al. 2006; Kumari et al. 2003; Lawrence et al. 2002; Thiel and Fink 2008; Thiel et al. 2005). In agreement with a "bottom up" role for nicotine, the drug's pro-cognitive effects have been suggested to be in part due to its stimulus filtering properties (Adler et al. 1992; Friedman et al. 1974; Kassel 1997; Knott et al. 2009; Metherate et al. 2012).

In the present study, drug effects on task were significant in the left VPC. In contrast, many studies have that examined the VPC during attention tasks have found response to be right lateralized, and the right VPC is traditionally presented as a hub of the ventral attention network (Corbetta and Shulman 2002). The left VPC effect observed in this study may be due to its relatively unique task parameters. Because subjects are asked to focus attention on linguistic stimuli (i.e. numbers), the task may be demanding on the left parietal cortex, as the left hemisphere is preferentially active during semantic processing (Bookheimer 2002). Indeed, verbal working memory is associated with left VPC response (Ravizza et al. 2011). In contrast, another attention study using visual, non-word stimuli has reported nicotinic suppression of right parietal cortex activity during no-cue trials during a Posner attention paradigm (Thiel et al. 2005). It is possible that both left and right VPC are important for selective attention, with relative lateralization influenced by the nature of the task.

Differential modulation by nicotine during selective attention was also observed in the AC. Previous studies have observed recruitment of the AC during attention tasks (Orr and Weissman 2009; Weissman et al. 2005; Weissman et al. 2004). More specifically, the dorsal $\mathrm{AC}$ is involved in focusing attention towards relevant stimuli, whereas the ventral AC is involved in focusing attention away from irrelevant stimuli (Orr and Weissman 2009). In the present study, a significant interaction was found in the ventral AC but not the dorsal AC, suggesting that the nicotine's effects on attention-related $\mathrm{AC}$ response are related to ignoring distraction. AC involvement in the present study may also be due in part to the "go/no-go" nature of the task. The SART task requires subjects to stop prepotent responses to irregular, infrequently occurring stimuli, particularly during the "Random" version. The AC plays a major role in top-down management of response conflict, including motor response conflict, as typically occurs in tasks with high commission error rates (Botvinick et al. 2004).

Recruitment of the AC may therefore represent or facilitate top-down behavioral modulation or control of performance (Botvinick et al. 2004). Nicotinic modulation of AC is also consistent with previous fMRI studies that showed decreased response of the AC in healthy subjects and increased response in schizophrenia patients during a smooth pursuit eye movement task (Tanabe et al. 2006).

Although nicotinic modulation of hippocampal response was not predicted a priori, the observed effects in the area are not surprising based on its functions. One of these functions 
is to assist in context-dependent encoding, such that "features" (such as events, locations, places in time) can be "bound" into a coherent organizational framework in memory to facilitate future recall (Eichenbaum and Cohen 2014). Furthermore, the hippocampus is recruited when subjects are asked to selectively attend to stimulus features, thereby increasing the likelihood that subjects will recall that feature in a later memory test (Uncapher and Rugg 2009). It is possible that hippocampal recruitment after nicotine administration during the OrderedNoise condition is indicative of increased resource allocation towards processing sensory stimuli, such as the environmental noise presented in this study.

Although a significant drug $\times$ difficulty $\times$ distraction interaction was observed (as hypothesized), the directionality of the effects was more variable than predicted. Based on the "attention allocation" model proposed by Kassel (1997), we predicted that nicotine would increase response under non-demanding task conditions and decrease response as demands increased, yielding the significant interaction. The nature of the interaction, however, was either U or inverted U-shaped, depending on the brain region. In the VPC and $\mathrm{AC}$, for example, nicotine increased response during the least demanding condition (OrderedSilent), decreased response under moderately demanding conditions (OrderedNoise and RandomSilent), and slightly increased response under the most demanding condition (RandomNoise). These findings suggest that the relative influence of nicotine on excitatory, "signal enhancing" processes and inhibitory, "filtering" processes may not be a monotonically increasing function of task demands as initially supposed. Our results further suggest that the presence of a distracting stimulus does not necessitate recruitment of "filtering" processes, as nicotine decreased response (relative to placebo) in the VPC and AC during the OrderedNoise task. The mechanisms by which nicotine may modulate response are unclear, but are likely due to a relative shift between influences on excitatory and inhibitory neuronal processes. Furthermore, additional studies with larger sample sizes will be needed to verify these conclusions, as post-hoc tests did not reveal significant simple main effects (e.g. Nicotine > Placebo during OrderedSilent). Finally, we cannot rule out other task-specific influences on the direction of nicotine's effects. For example, Thiel et al. (2005) found that nicotine (slightly) increased parietal activity during valid cuing but decreased parietal activity during invalid cuing. This difference is more likely to be due to cortical processes evoked by attention shifting during invalid cuing rather than a general increase or decrease in attentional demand.

Consistent with our findings of a significant dependence of task conditions (difficulty and distraction) on the effects of nicotine, previous fMRI studies using a variety of paradigms have shown both increased and decreased task-associated brain response to the drug (Hahn et al. 2009; Hahn et al. 2007; Kumari et al. 2003; Lawrence et al. 2002; Thiel and Fink 2008; Thiel et al. 2005); reviewed by Newhouse et al. (2011). One fMRI study that examined selective attention, by Hahn et al. (2009), used a visual task in which subjects were asked to differentiate between a set of features (e.g. color) while ignoring a second set of features. Comparing smokers and nonsmokers at baseline, Hahn et al. found that smokers showed decreased response under demanding conditions and increased response under nondemanding task conditions. After nicotine administration in smokers, response was 
normalized, consistent with the hypothesis that nicotine may help "optimize" the level of neuronal activity.

As with any drug study, task-dependent modulation of a particular area does not necessarily imply that a drug is binding to its receptor target in that area. In the human cortex, nicotinic receptors are most highly expressed in the parietal, somatosensory, and motor cortices, with lower levels of expression in prefrontal and cingulate cortices and hippocampus (Paterson and Nordberg 2000; Sihver et al. 1998). The a7 receptor subtype also demonstrates moderate levels of expression in the hippocampus (Paterson and Nordberg 2000). It is therefore likely that, to some extent, the observed results can be explained by nicotinic receptor binding directly to receptors in the hippocampus, AC and VPC. fMRI techniques do not currently allow researchers to distinguish between "primary" brain effects (due to local nicotinic receptor activation) and "secondary" effects (which arise as a consequence of primary effects independent of nicotine binding). The finding, however, that the main effect of nicotine differed substantially from interaction effects (with response differences only in the motor and somatosensory cortices) suggests that the study parameters (e.g. noise distraction) were primarily responsible for the observed effects. It is similarly unlikely that the observed results are due to a nonspecific effect of nicotine on BOLD response, given that 1) nicotine's effects on BOLD response are highly variable between subjects (Warbrick et al. 2011),2) nicotine did not significantly affect blood pressure or heart rate in this study, and 3) the main effect of nicotine differed from the interaction effects.

Although we observed significant functional effects of nicotine in the present study, surprisingly, we did not observe significant interaction effects of the drug on any behavioral performance measure. This negative finding may be due to the possibility that 1 ) performance metrics such as commission errors are reliant on other processes (e.g. response inhibition) that are relatively insensitive to nicotinic modulation, 2) a ceiling effect on task performance has already been reached, or 3) neuronal response differences may in part represent re-allocation of resources associated with other "tasks" for which performance was not measured in the present study (e.g. mind wandering, divided attention). Clinical populations of subjects that have reduced baseline levels of nicotinic signaling as well as attentional dysfunction, such as patients with schizophrenia (Freedman 2014), may show stronger behavioral effects of the drug. It is also possible that the study is underpowered to observe behavioral effects. Indeed, changes in neuronal response observed with fMRI are usually more sensitive to pharmacologic modulation than behavioral metrics (Newhouse et al. 2011).

\section{Conclusion}

Although behavioral and electrophysiological studies have demonstrated that nicotine improves selective attention and enhances its associated neurophysiological features, to our knowledge this is the first study that has used fMRI to examine the effects of nicotine administration during auditory distraction in the healthy human nonsmokers. Our results suggest that nicotine may differentially affect response in the hippocampus, VPC and AC depending on task difficulty and distraction level. Future studies may examine how these results interact with the neuronal effects of nicotine in clinical populations. 


\section{Acknowledgments}

The authors thank Debra Singel for assistance with data acquisition. This work was supported by NIMH Conte Center Grant MH-086383, the VA Biomedical Laboratory and Clinical Science Research and Development Service, the Brain and Behavior Foundation, and the Blowitz-Ridgeway Foundation.

\section{References}

Adler LE, Hoffer LJ, Griffith J, Waldo MC, Freedman R. Normalization by nicotine of deficient auditory sensory gating in the relatives of schizophrenics. Biol Psychiatry. 1992; 32:607-616. [PubMed: 1450287]

Anbeek P, Vincken KL, van Bochove GS, van Osch MJ, van der Grond J. Probabilistic segmentation of brain tissue in MR imaging. Neuroimage. 2005; 27:795-804. [PubMed: 16019235]

Bain JN, Prendergast MA, Terry AV Jr, Arneric SP, Smith MA, Buccafusco JJ. Enhanced attention in rhesus monkeys as a common factor for the cognitive effects of drugs with abuse potential. Psychopharmacology (Berl). 2003; 169:150-160. [PubMed: 12768267]

Bookheimer S. Functional MRI of language: new approaches to understanding the cortical organization of semantic processing. Annu Rev Neurosci. 2002; 25:151-188. [PubMed: 12052907]

Botvinick MM, Cohen JD, Carter CS. Conflict monitoring and anterior cingulate cortex: an update. Trends Cogn Sci. 2004; 8:539-546. [PubMed: 15556023]

Childress A, Sallee FR. Pozanicline for the treatment of attention-deficit/hyperactivity disorder. Expert Opin Investig Drugs. 2014; 23:1585-1593.

Corbetta M, Shulman GL. Control of goal-directed and stimulus-driven attention in the brain. Nat Rev Neurosci. 2002; 3:201-215. [PubMed: 11994752]

Corbetta M, Shulman GL. Spatial neglect and attention networks. Annu Rev Neurosci. 2011; 34:569599. [PubMed: 21692662]

Demeter E, Sarter M. Leveraging the cortical cholinergic system to enhance attention. Neuropharmacology. 2013; 64:294-304. [PubMed: 22796110]

Edmister WB, Talavage TM, Ledden PJ, Weisskoff RM. Improved auditory cortex imaging using clustered volume acquisitions. Hum Brain Mapp. 1999; 7:89-97. [PubMed: 9950066]

Eichenbaum H, Cohen NJ. Can we reconcile the declarative memory and spatial navigation views on hippocampal function? Neuron. 2014; 83:764-770. [PubMed: 25144874]

Eysenck, MW. Attention and Arousal: Cognition and Performance. Springer-Verlag; New York: 1982.

Fleisher C, McGough J. Sofinicline: a novel nicotinic acetylcholine receptor agonist in the treatment of attention-deficit/hyperactivity disorder. Expert Opin Investig Drugs. 2014; 23:1157-1163.

Freedman R. alpha7-nicotinic acetylcholine receptor agonists for cognitive enhancement in schizophrenia. Annu Rev Med. 2014; 65:245-261. [PubMed: 24111888]

Friedman J, Horvath T, Meares R. Tobacco smoking and a 'stimulus barrier'. Nature. 1974; 248:455456. [PubMed: 4823676]

Ghaleiha A, Ghyasvand M, Mohammadi MR, Farokhnia M, Yadegari N, Tabrizi M, Hajiaghaee R, Yekehtaz H, Akhondzadeh S. Galantamine efficacy and tolerability as an augmentative therapy in autistic children: A randomized, double-blind, placebo-controlled trial. J Psychopharmacol. 2013; 28:677-685. [PubMed: 24132248]

Giessing C, Thiel CM, Rosler F, Fink GR. The modulatory effects of nicotine on parietal cortex activity in a cued target detection task depend on cue reliability. Neuroscience. 2006; 137:853864. [PubMed: 16309846]

Gilbert DG, Sugai C, Zuo Y, Rabinovich NE, McClernon FJ, Froeliger B. Brain indices of nicotine's effects on attentional bias to smoking and emotional pictures and to task-relevant targets. Nicotine Tob Res. 2007; 9:351-363. [PubMed: 17365767]

Grobe JE, Perkins KA, Goettler-Good J, Wilson A. Importance of environmental distractors in the effects of nicotine on short-term memory. Exp Clin Psychopharmacol. 1998; 6:209-216. [PubMed: 9608353] 
Hahn B, Ross TJ, Wolkenberg FA, Shakleya DM, Huestis MA, Stein EA. Performance effects of nicotine during selective attention, divided attention, and simple stimulus detection: an fMRI study. Cereb Cortex. 2009; 19:1990-2000. [PubMed: 19073624]

Hahn B, Ross TJ, Yang Y, Kim I, Huestis MA, Stein EA. Nicotine enhances visuospatial attention by deactivating areas of the resting brain default network. J Neurosci. 2007; 27:3477-3489. [PubMed: 17392464]

Hahn B, Shoaib M, Stolerman IP. Nicotine-induced enhancement of attention in the five-choice serial reaction time task: the influence of task demands. Psychopharmacology (Berl). 2002; 162:129_ 137. [PubMed: 12110990]

Howe WM, Ji J, Parikh V, Williams S, Mocaer E, Trocme-Thibierge C, Sarter M. Enhancement of attentional performance by selective stimulation of alpha4beta2 $(*)$ nAChRs: underlying cholinergic mechanisms. Neuropsychopharmacology. 2010; 35:1391-1401. [PubMed: 20147893]

Kassel JD. Smoking and attention: a review and reformulation of the stimulus-filter hypothesis. Clin Psychol Rev. 1997; 17:451-478. [PubMed: 9260037]

Knott VJ, Bolton K, Heenan A, Shah D, Fisher DJ, Villeneuve C. Effects of acute nicotine on eventrelated potential and performance indices of auditory distraction in nonsmokers. Nicotine Tob Res. 2009; 11:519-530. [PubMed: 19380382]

Kriegeskorte N, Simmons WK, Bellgowan PS, Baker CI. Circular analysis in systems neuroscience: the dangers of double dipping. Nat Neurosci. 2009; 12:535-540. [PubMed: 19396166]

Kumari V, Gray JA, ffytche DH, Mitterschiffthaler MT, Das M, Zachariah E, Vythelingum GN, Williams SC, Simmons A, Sharma T. Cognitive effects of nicotine in humans: an fMRI study. Neuroimage. 2003; 19:1002-1013. [PubMed: 12880828]

Lawrence NS, Ross TJ, Stein EA. Cognitive mechanisms of nicotine on visual attention. Neuron. 2002; 36:539-548. [PubMed: 12408855]

Levin ED, Conners CK, Silva D, Hinton SC, Meck WH, March J, Rose JE. Transdermal nicotine effects on attention. Psychopharmacology (Berl). 1998; 140:135-141. [PubMed: 9860103]

Metherate R, Intskirveli I, Kawai HD. Nicotinic filtering of sensory processing in auditory cortex. Front Behav Neurosci. 2012; 6:44. [PubMed: 22833720]

Newhouse PA, Potter A, Singh A. Effects of nicotinic stimulation on cognitive performance. Curr Opin Pharmacol. 2004; 4:36-46. [PubMed: 15018837]

Newhouse PA, Potter AS, Dumas JA, Thiel CM. Functional brain imaging of nicotinic effects on higher cognitive processes. Biochem Pharmacol. 2011; 82:943-951. [PubMed: 21684262]

Orr JM, Weissman DH. Anterior cingulate cortex makes 2 contributions to minimizing distraction. Cereb Cortex. 2009; 19:703-711. [PubMed: 18653665]

Paterson D, Nordberg A. Neuronal nicotinic receptors in the human brain. Prog Neurobiol. 2000; 61:75-111. [PubMed: 10759066]

Poltavski DV, Petros T. Effects of transdermal nicotine on prose memory and attention in smokers and nonsmokers. Physiol Behav. 2005; 83:833-843. [PubMed: 15639169]

Poltavski DV, Petros T. Effects of transdermal nicotine on attention in adult non-smokers with and without attentional deficits. Physiol Behav. 2006; 87:614-624. [PubMed: 16466655]

Provost SC, Woodward R. Effects of nicotine gum on repeated administration of the Stroop test. Psychopharmacology (Berl). 1991; 104:536-540. [PubMed: 1780425]

Ravizza SM, Hazeltine E, Ruiz S, Zhu DC. Left TPJ activity in verbal working memory: implications for storage- and sensory-specific models of short term memory. Neuroimage. 2011; 55:18361846. [PubMed: 21168512]

Seli P, Cheyne JA, Barton KR, Smilek D. Consistency of sustained attention across modalities: comparing visual and auditory versions of the SART. Can J Exp Psychol. 2012; 66:44-50. [PubMed: 21910522]

Sihver W, Gillberg PG, Nordberg A. Laminar distribution of nicotinic receptor subtypes in human cerebral cortex as determined by $[3 \mathrm{H}](-)$ nicotine, $[3 \mathrm{H}]$ cytisine and $[3 \mathrm{H}]$ epibatidine in vitro autoradiography. Neuroscience. 1998; 85:1121-1133. [PubMed: 9681951]

Smucny J, Rojas DC, Eichman LC, Tregellas JR. Neuronal effects of auditory distraction on visual attention. Brain Cogn. 2013; 81:263-270. [PubMed: 23291265] 
Stolerman IP, Mirza NR, Shoaib M. Nicotine psychopharmacology: addiction, cognition and neuroadaptation. Med Res Rev. 1995; 15:47-72. [PubMed: 7898169]

Tanabe J, Tregellas JR, Martin LF, Freedman R. Effects of nicotine on hippocampal and cingulate activity during smooth pursuit eye movement in schizophrenia. Biol Psychiatry. 2006; 59:754761. [PubMed: 16259965]

Thiel CM, Fink GR. Effects of the cholinergic agonist nicotine on reorienting of visual spatial attention and top-down attentional control. Neuroscience. 2008; 152:381-390. [PubMed: 18272290]

Thiel CM, Zilles K, Fink GR. Nicotine modulates reorienting of visuospatial attention and neural activity in human parietal cortex. Neuropsychopharmacology. 2005; 30:810-820. [PubMed: 15668726]

Tregellas JR, Ellis J, Shatti S, Du YP, Rojas DC. Increased hippocampal, thalamic, and prefrontal hemodynamic response to an urban noise stimulus in schizophrenia. Am J Psychiatry. 2009; 166:354-360. [PubMed: 19147695]

Uncapher MR, Rugg MD. Selecting for memory? The influence of selective attention on the mnemonic binding of contextual information. J Neurosci. 2009; 29:8270-8279. [PubMed: 19553466]

Valles AS, Borroni MV, Barrantes FJ. Targeting Brain alpha7 Nicotinic Acetylcholine Receptors in Alzheimer's Disease: Rationale and Current Status. CNS Drugs. 2014

Warbrick T, Mobascher A, Brinkmeyer J, Musso F, Stoecker T, Shah NJ, Vossel S, Winterer G. Direction and magnitude of nicotine effects on the fMRI BOLD response are related to nicotine effects on behavioral performance. Psychopharmacology (Berl). 2011; 215:333-344. [PubMed: 21243486]

Weissman DH, Gopalakrishnan A, Hazlett CJ, Woldorff MG. Dorsal anterior cingulate cortex resolves conflict from distracting stimuli by boosting attention toward relevant events. Cereb Cortex. 2005; 15:229-237. [PubMed: 15238434]

Weissman DH, Warner LM, Woldorff MG. The neural mechanisms for minimizing cross-modal distraction. J Neurosci. 2004; 24:10941-10949. [PubMed: 15574744]

Yu LF, Zhang HK, Caldarone BJ, Eaton JB, Lukas RJ, Kozikowski AP. Recent Developments in Novel Antidepressants Targeting alpha4beta2-Nicotinic Acetylcholine Receptors. J Med Chem. 2014; 57:8204-8223. [PubMed: 24901260] 

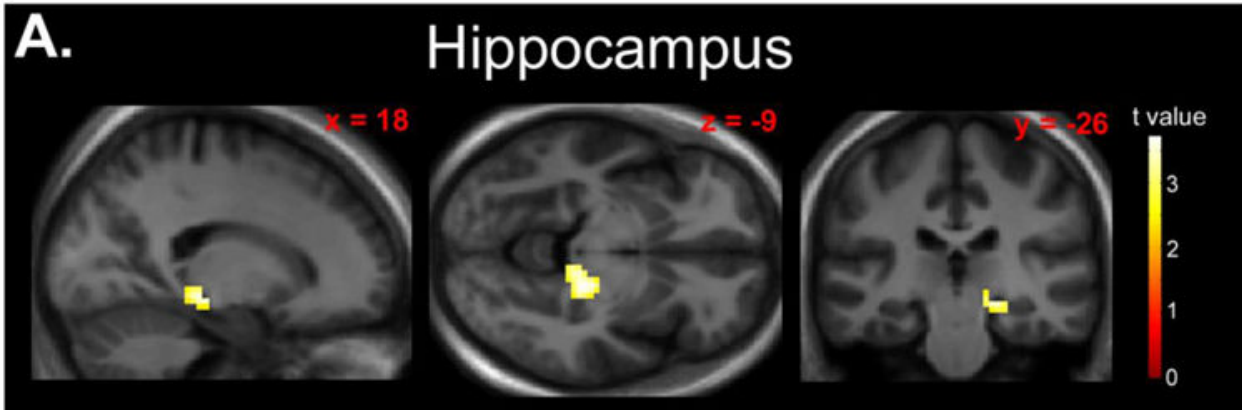

B.

BOLD Response, All Task Conditions

C. Effect of Nicotine on
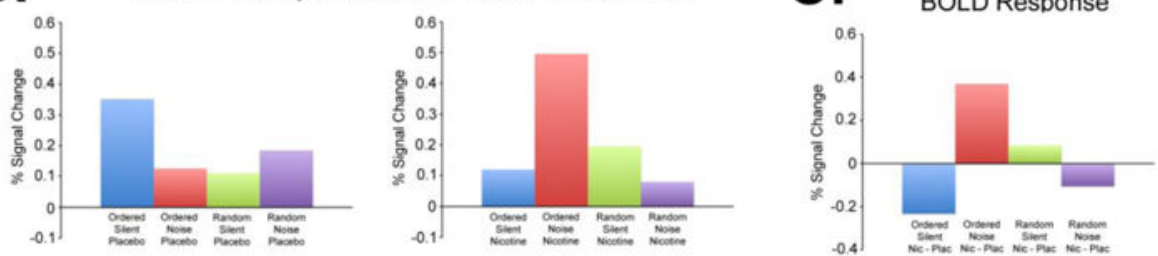

Fig 1.

A) Statistical parametric map displaying a significant drug $\times$ difficulty $\times$ distraction interaction effect in the right hippocampus. Map thresholded at $\mathrm{p}<0.01$, cluster size 62 voxels. Images are displayed in the neurologic convention ( $\mathrm{R}$ on $\mathrm{R}$ ). B) Plotted peak BOLD responses (\% signal changes) for each experimental condition (relative to fixation baseline). C) Plotted peak BOLD responses (\% signal changes) for each condition collapsed across the effect of nicotine (Nicotine > Placebo) displaying the nature of the significant interaction. 
A. Ventral Parietal Cortex
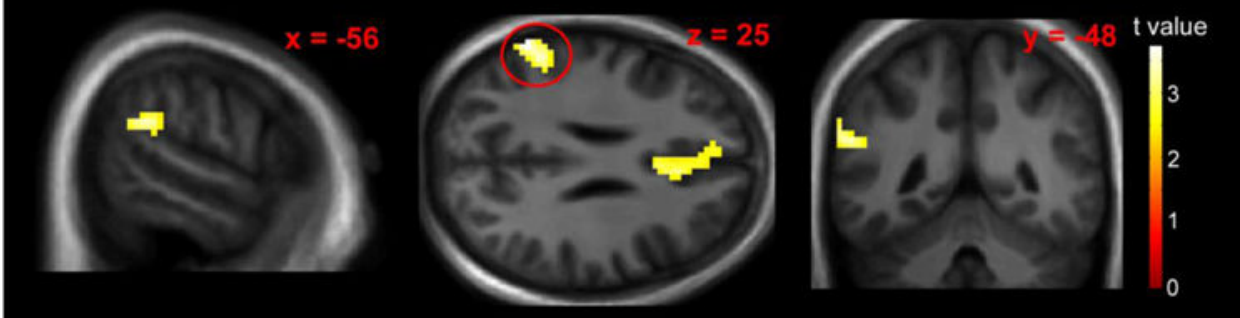

B.

BOLD Response, All Task Conditions

C. Effect of Nicotine on
BOLD Response
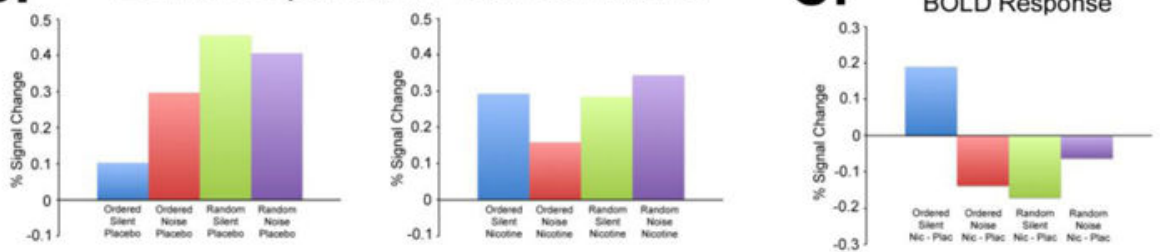

Fig. 2.

A) Statistical parametric map displaying a significant drug $\times$ difficulty $\times$ distraction interaction effect in the left VPC. Map thresholded at $\mathrm{p}<0.01$, cluster size 62 voxels. Images are displayed in the neurologic convention ( $\mathrm{R}$ on $\mathrm{R})$. B) Plotted peak BOLD responses (\% signal changes) for each experimental condition (relative to fixation baseline). C) Plotted peak BOLD responses (\% signal changes) for each condition collapsed across the effect of nicotine (Nicotine > Placebo) displaying the nature of the significant interaction. 
A. Anterior Cingulate

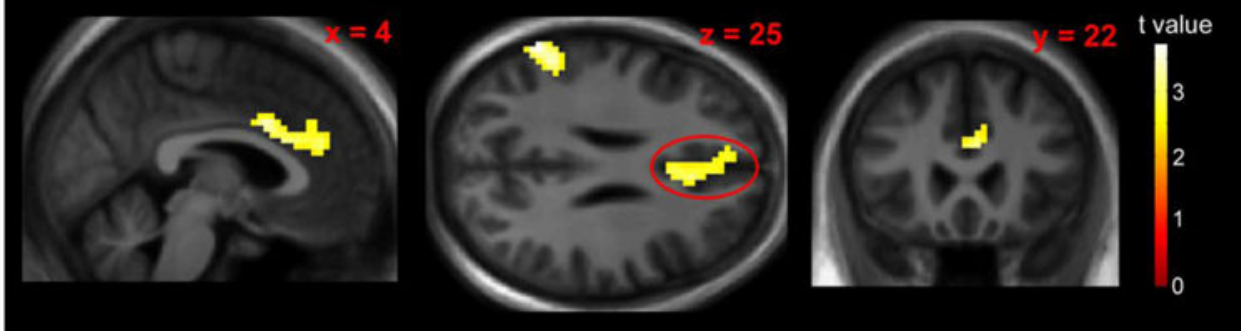

B. BOLD Response, All Task Conditions
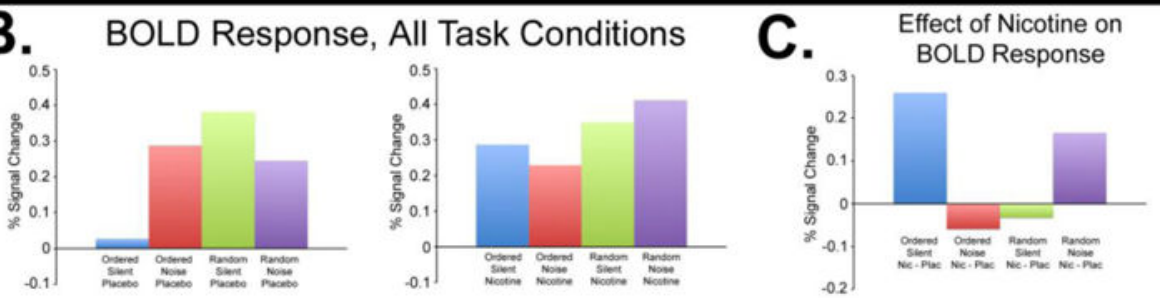

Fig. 3.

A) Statistical parametric map displaying a significant drug $\times$ difficulty $\times$ distraction interaction effect in the AC. Map thresholded at $p<0.01$, cluster size 62 voxels. Images are displayed in the neurologic convention ( $\mathrm{R}$ on $\mathrm{R}$ ). B) Plotted peak BOLD responses (\% signal changes) for each experimental condition (relative to fixation baseline). C) Plotted peak BOLD responses (\% signal changes) for each condition collapsed across the effect of nicotine (Nicotine > Placebo) displaying the nature of the significant interaction. 


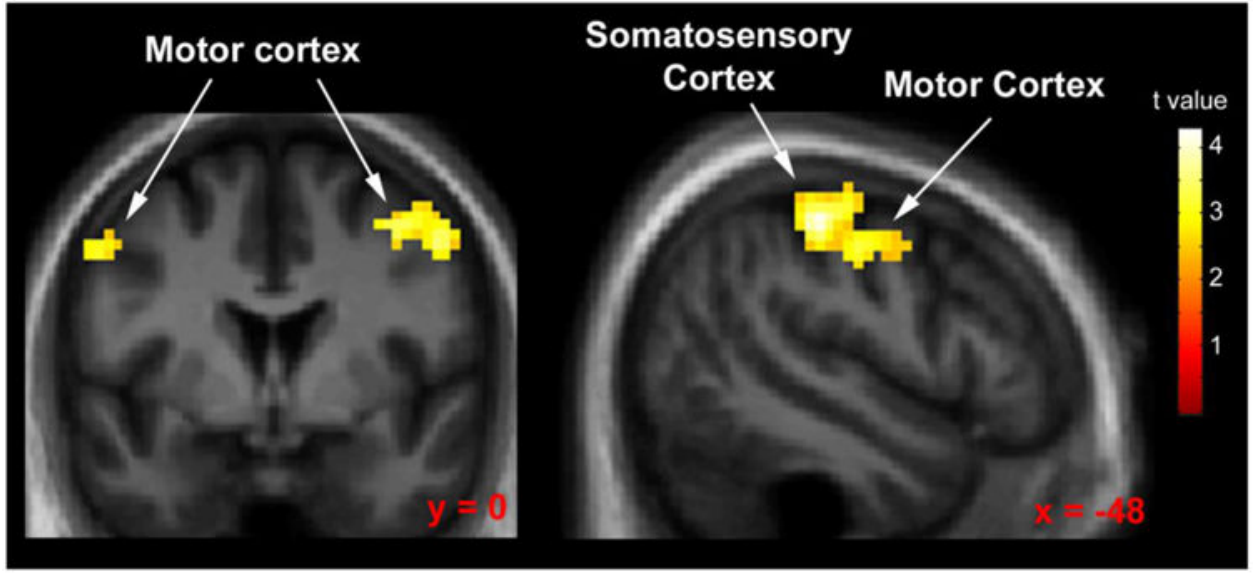

Fig 4.

Statistical parametric map displaying areas of significantly increased response during nicotine administration (relative to placebo) collapsed across all other task conditions. Map thresholded at $\mathrm{p}<0.01$, cluster size 62 voxels. Images are displayed in the neurologic convention ( $\mathrm{R}$ on $\mathrm{R})$. 
Table 1

Physiological effects of placebo and $7 \mathrm{mg}$ nicotine patch

\pm symbols represent the standard error of the mean.

\begin{tabular}{lcccc}
\hline Measure & Placebo, Pretreatment & $\begin{array}{l}\text { Placebo, 60 m } \\
\text { Posttreatment }\end{array}$ & Nicotine, Pretreatment & $\begin{array}{c}\text { Nicotine, 60 m } \\
\text { Posttreatment }\end{array}$ \\
Systolic BP (mmHg) & $128 \pm 3.83$ & $121 \pm 3.28$ & $127 \pm 3.05$ & $125 \pm 2.36$ \\
Diastolic BP (mmHg) & $79.0 \pm 1.97$ & $77.2 \pm 2.20$ & $78.9 \pm 1.76$ & $79.7 \pm 1.84$ \\
Heart rate (bpm) & $74.8 \pm 2.66$ & $72.8 \pm 3.16$ & $76.4 \pm 2.65$ & $77.3 \pm 2.85$ \\
\hline
\end{tabular}

Abbreviations: $\mathrm{BP}=$ blood pressure, $\mathrm{mmHg}=\mathrm{mm}$ of mercury, $\mathrm{bpm}=$ beats per minute. 


\section{Table 2}

\section{a. Behavioral Data, Ordered SART}

\pm symbols represent the standard error of the mean.

\begin{tabular}{lccc}
\hline Measure & Drug & Ordered Silent & Ordered Noise \\
$\%$ Errors of Commission & Placebo & $5.66 \pm 1.31$ & $5.45 \pm 1.50$ \\
& Nicotine & $5.64 \pm 1.59$ & $5.68 \pm 1.78$ \\
$\%$ Errors of Omission & Placebo & $1.60 \pm 0.71$ & $2.49 \pm 0.90$ \\
& Nicotine & $2.02 \pm 0.97$ & $3.45 \pm 1.31$ \\
Reaction Time & Placebo & $433 \pm 24.0$ & $447 \pm 24.1$ \\
& Nicotine & $425 \pm 24.3$ & $441 \pm 26.0$ \\
\hline
\end{tabular}

b. Behavioral Data, Random SART. \pm symbols represent the standard error of the mean.

\begin{tabular}{lccc} 
Measure & Drug & Random Silent & Random Noise \\
\% Errors of Commission & Placebo & $16.8 \pm 3.45$ & $19.5 \pm 4.76$ \\
& Nicotine & $15.3 \pm 2.61$ & $19.5 \pm 3.72$ \\
\% Errors of Omission & Placebo & $0.875 \pm 0.250$ & $4.14 \pm 0.91$ \\
& Nicotine & $1.19 \pm 0.45$ & $5.33 \pm 2.00$ \\
Reaction Time & Placebo & $540 \pm 16.2$ & $559 \pm 17.9$ \\
& Nicotine & $543 \pm 19.0$ & $563 \pm 20.7$ \\
\hline
\end{tabular}




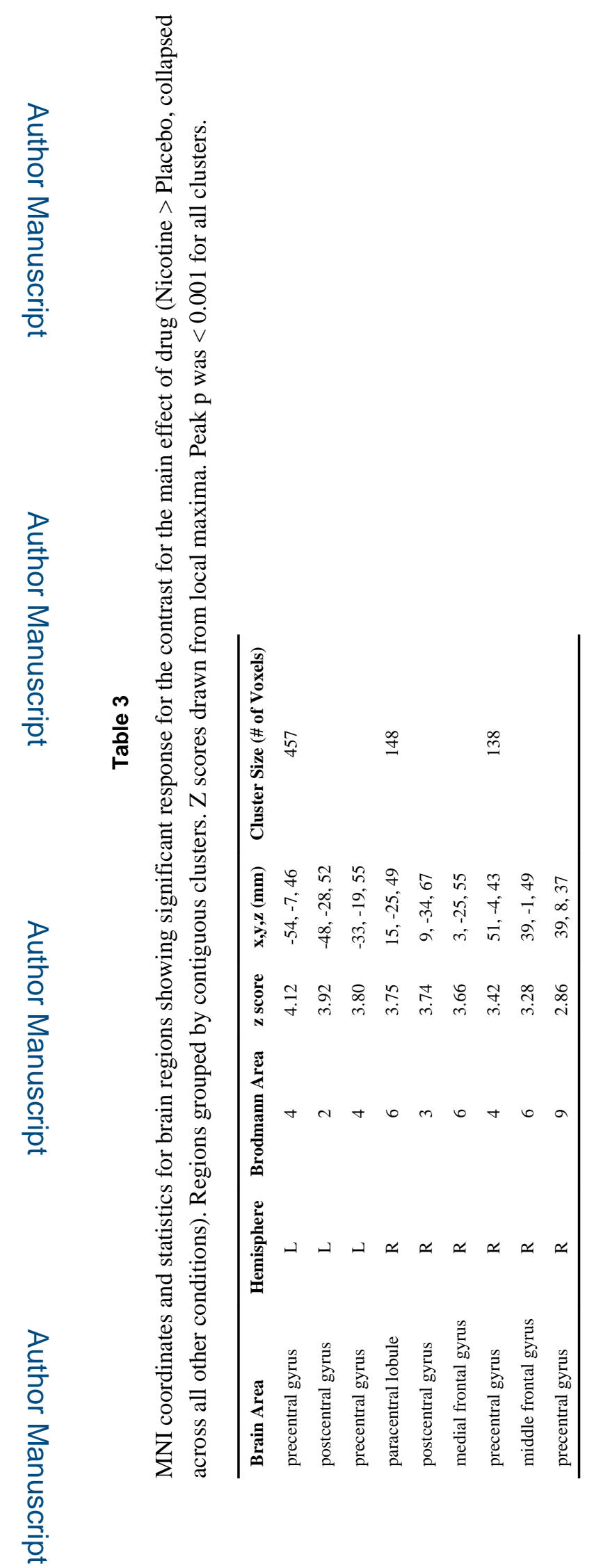

Psychopharmacology (Berl). Author manuscript; available in PMC 2016 June 01. 


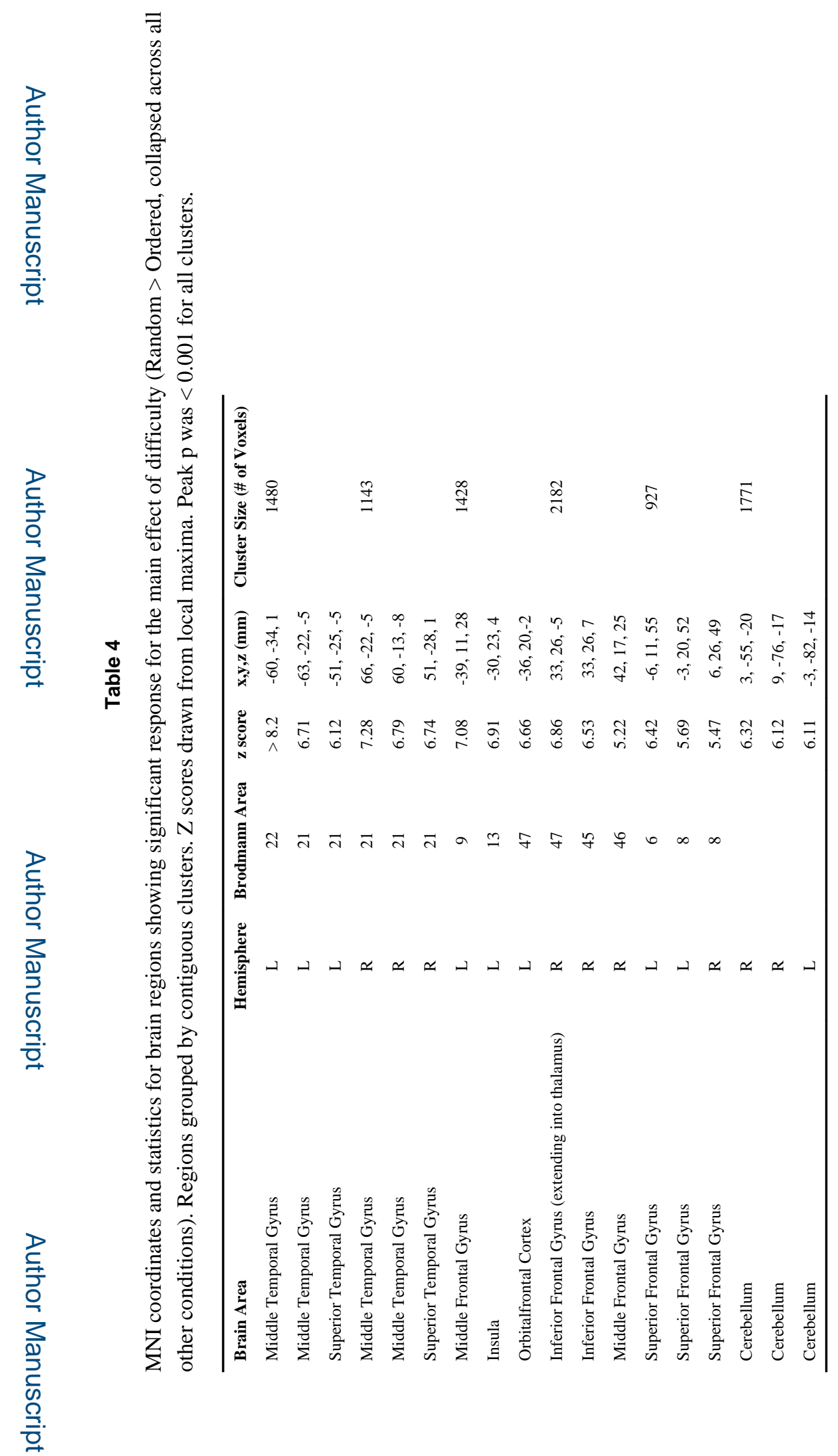

Psychopharmacology (Berl). Author manuscript; available in PMC 2016 June 01. 


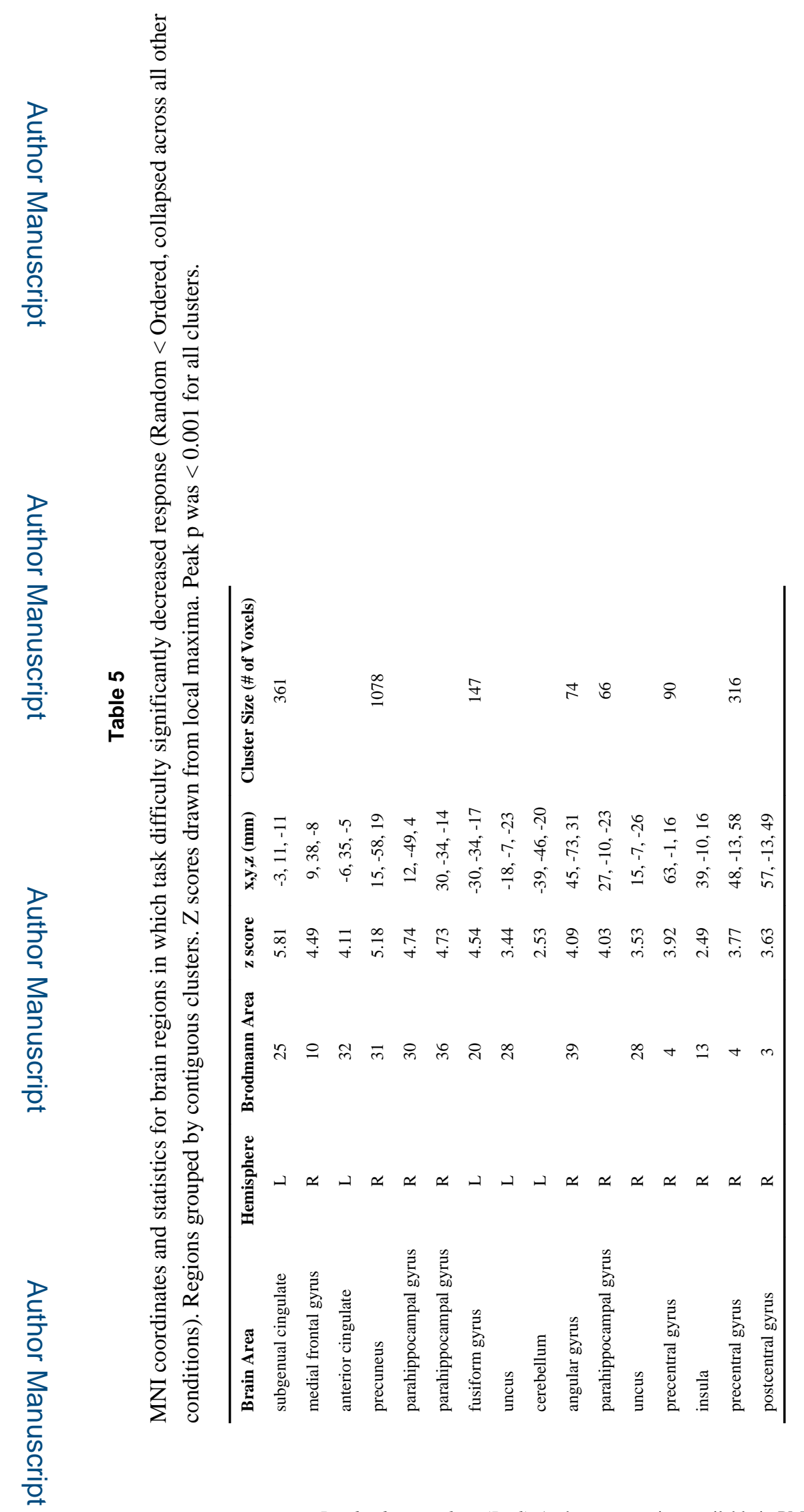

Psychopharmacology (Berl). Author manuscript; available in PMC 2016 June 01. 